the median duration of disease 2 years) selected according to 2010 ACR/EULAR diagnostic criteria were included. 30 healthy control groups were selected. Anti carP antibody Anti-carbamylated Protein Human anti-carbamylated Protein Antibody (ACP-Ab) ELISA Kit (SunRedBio, China) was used for measurement of antibodies against carbamylated proteins.

Results: The study population consisted of 133 subjects, 30 controls, 57 SLEs and 46 RAs. The mean age of SLE patients was lower than that of RA patients. $(40,9 \pm 13,7$ versus $54,2 \pm 12,4 ; p<0,001)$. The proportion of active smokers was found to be higher in RA patients compared to SLE patients (19.6\% versus $5.3 \%$, $\mathrm{p}=0.005)$. The frequency of anti carP antibody positivity was $3.3 \%$ in the healthy control group. In contrast, the frequency of anti carP antibody positivity was found as high as $17.4 \%$ in patients with RA. $(p=<0.001)$, And this frequency was $54.4 \%$ in the SLE patient group $(\mathrm{p}=<0.001)$ Anti carP antibody predicted SLE patients with $54.4 \%$ sensitivity and $96.7 \%$ specificity compared to the healthy control group. (AUC: $0.755, \mathrm{p}<0.001$ ) Anti carP antibody predicted RA patients with $17.4 \%$ sensitivity and $96.7 \%$ specificity compared to the healthy control group (AUC: $0.570, p=0.032$ ). Anti carP antibody predicted SLE patients with $54.5 \%$ sensitivity and $82.6 \%$ specificity compared to healthy RA group (AUC: 0.685 , $\mathrm{p}<0.001)$. AnticarP antibodies were found to be positive in all of the SLE patient groups with anti-CCP positivity. There was no significant difference in terms of in organ involvement between anti-carp antibody positive or negative SLE patients. Anti-carP antibody positivity was assessed by ROC Curve analysis for the prediction of diagnostic performance in SLE patients compared to RA patients. Accordingly, Anti carP antibody positivity, ANA positivity, were found to have similar diagnostic performance. (AUC: 0.639)

Abstract THU0345 - Table 1. Anti carbamylated protein antibody positivity distribution across groups

\begin{tabular}{|c|c|c|c|c|}
\hline Variables & $\begin{array}{c}\text { control } \\
n=30\end{array}$ & $\begin{array}{c}\text { SLE } \\
n=57\end{array}$ & $\begin{array}{c}\mathrm{RA} \\
\mathrm{n}=46\end{array}$ & $\mathrm{p}$ \\
\hline \multicolumn{5}{|c|}{ AnticarP antibody } \\
\hline Negative & $29(96,7)$ & $26(45,6)$ & $38(82,6)$ & $<0,001$ \\
\hline Pozitive & $1(3,3)$ & $31(54,4)$ & $8(17,4)$ & \\
\hline
\end{tabular}

SLE:systemic lupus erythematosus RA: rheumatoid arthritis)

Conclusions: Antibody positivity was found to be $54.4 \%$ in SLE patient group. It is significantly higher in SLE compared to healthy control and RA patient group. In the SLE group, it is still a more significant diagnostic prognostic than the healthy control and RA group. Both SLE and RA patients have significant sensitivity and specificity compared to the healthy control group.

Disclosure of Interest: None declared

DOI: 10.1136/annrheumdis-2018-eular.4882

\section{THU0346 DETERMINANTS OF SONOGRAPHIC GLANDULAR DAMAGE IN A LARGE COHORT OF PATIENTS WITH PRIMARY SJÖGREN'S SYNDROME AND ITS IMPACT ON SALIVARY GLAND DYSFUNCTION}

${ }^{1}$ C. Baldini, N. Luciano ${ }^{1}$, F. Ferro ${ }^{1}$, E. Calabresi ${ }^{1}$, E. Elefante ${ }^{1}$, V. Donati ${ }^{2}$, S. Bombardieri ${ }^{1}$, M. Mosca ${ }^{1} .{ }^{1}$ Rheumatology Unit, University of Pisa; ${ }^{2}$ Pathologic Anatomy II, AOUP, Pisa, Italy

Background: Salivary gland ultrasonography (SGUS) has increasingly appeared as a valid tool to characterise major salivary glands involvement in primary Sjögren's syndrome (pSS). An international score based on the number and location of the hypoechoic areas in the gland has been proposed for diagnostic purposes. Recently, a specific interest in using SGUS to estimate major salivary gland damage has also arisen in order to individualise patients treatment.

Objectives: a) to investigate frequency and severity of salivary gland damage in a large monocentric cohort of pSS patients; b) to explore determinants that could be associated with salivary gland damage accrual.

Methods: Sonographic data from a monocentric cohort of 311 pSS patients were collected from 2012 to 2017 . SGUS had been performed according to a standard protocol that evaluated the echostructure of each gland graded on a 5-point scale (0-4). Salivary gland damage was defined by the presence of hyperechoic bands in more than $50 \%$ of the glandular parenchyma and on the basis of the size of the four glands.

Results: We included in the study 311 patients. The median age of the patients was 58 years (IQR, 49-68) and the median disease duration was 3 years (IQR, $0-9)$. Out of the cohort, $163(52.4 \%)$ patients underwent SGUS examination within 3 years from the diagnosis, 91 (29.3\%) within 4 to 10 years and $57(18.3 \%) 10$ years or more after the diagnosis. At SGUS evaluation 134/311 (43.1\%) pSS patients did not present any findings of damage, whereas 177/311 (56.9\%) presented at least one element of damage. SGUS damage inversely correlated with USFR $(r=-0.382$, $p$-value $=0.001)$. At the univariate analysis SGUS damage was associated to disease duration, number and location of the hypo-anechoic areas, focus score, ESSDAI score, hypergammaglobulinemia, and positivity for anti-Ro/ SSA, anti-La/SSB, Rheumatoid Factor (RF) and cryoglobulins. In the linear regression, duration of the follow-up, number and location of the hypo-anechoic areas and presence of cryoglobulins remained independently associated with SGUS damage. In 138/311 (44.4\%) patients, SGUS was repeated prospectively at least twice during a median follow-up of 24 months (IQR, 12-36). Notably, 23 $138(16.7 \%)$ patients presented a damage progression. Presence of hypere choich bands in the submandibular glands at the baseline evaluation was an independent risk factor for damage progression, whereas pSS low disease activity (ESSDAI <5) was protective against damage accrual.

Conclusions: Ultrasonographic damage resulted quite frequent in pSS patients and significantly associated with salivary gland dysfunction. Notably, pSS patients with normal echostructure of their glands were those less prone to develop SGUS damage during the disease course, whereas those presenting localised, diffuse or scattered hypo-anechoic areas were at higher risk of damage accrual.

Disclosure of Interest: None declared

DOI: 10.1136/annrheumdis-2018-eular.5843

\section{THU0347 AQUAPORIN-4 IMMUNOGLOBULIN G ANTIBODY POSITIVE NEUROMYELITIS OPTICA SPECTRUM DISORDER AND SYSTEMIC AUTOIMMUNE DISEASES OVERLAP SYNDROME: A SINGLE CENTREEXPERIENCE}

E. Martin Nares, G. Hernández Molina, H. Fragoso Loyo. Immunology and Rheumatology Department, Instituto Nacional de Ciencias Médicas y Nutrición Salvador Zubirán, Mexico City, Mexico

Background: The coexistence of neuromyelitis optica spectrum disorder (NMOSD) with other systemic autoimmune diseases is well recognised, especially with systemic lupus erythematosus (SLE) and Sjögren syndrome (SS). However literature is scarce, limited to case reports and multicentric case series.

Objectives: To describe the clinical and radiological characteristics and out comes of patients with AQP4-IgG seropositive NMOSD coexisting with SLE and SS in a single centre.

Methods: This was a retrospective study that included patients with concurrent diagnosis of AQP4-lgG seropositive NMOSD according to the 2015 Internationa Consensus Diagnostic Criteria, and SLE according to the ACR revised criteria or SS according to the AECG criteria who regularly attended a tertiary referral centre in Mexico City (2003-2018). We collected demographics, clinical (neurological events, number of relapses, remission, treatment, follow-up [date of last visit to a rheumatologist and/or neurologist] and disability according to the Expanded Disability Status Scale [EDSS]), laboratory (cerebrospinal fluid (CSF) analysis) and imaging data of NMOSD, as well as clinical and serological data of the overlapping autoimmune disease. We assessed disease activity in SLE and SS using SLE DAI-2K and ESSDAI respectively, and accrual damage with the SLICC/ACR-DI and SSDDI respectively.

Results: We included 11 patients, 10 (90.9\%) women with a mean age at diagnosis of $36 \pm 15$ years. Seven $(63.6 \%)$ had SLE and $4(36.6 \%)$ primary SS. Five $(45.5 \%)$ patients had also another systemic or organ-specific autoimmune dis ease. In $8(72.7 \%)$ patients NMOSD followed SLE/SS onset, $3(27.3 \%)$ had a simultaneous presentation, and in $1(9.1 \%)$ NMOSD preceded SS diagnosis. The mean time from diagnosis of SLE/SS to the first neurological event was 54.6 months. The mean SLEDAI-2K and ESSDAI at first neurological event was 3.1 (mainly hypocomplemetemia and high anti-dsDNA) and 14.3 points (mainly rena and peripheral nerve involvement) respectively. During follow-up, 10 patients $(90.9 \%)$ experienced myelitis, 5 (45.5\%) optic neuritis, 2 (18.2\%) each experienced area postrema syndrome, acute brainstem syndrome and cerebral syndrome; being the median number of neurological events $4 .^{1-8}$ Three patients $(27.3 \%)$ had antiphospholipid antibodies. None of the patients had pleocytosis or low CSF glucose and 3 had high CSF proteins. All patients had longitudinally extensive transverse myelitis on MRI, $3(27.3 \%)$ optic nerve findings and 6 $(54.5 \%)$ NMOSD-typical brain lesion patterns. Nine $(81.8 \%)$ patients went into either total or partial NMOSD remission at a mean follow up of $6.5 \pm 5.3$ years. At last follow up the median EDSS, SLICC/ACR-DI and SSDDI was 2.5 (1-10), 2 (0$7)$ and $2(0-3)$ points respectively; 4 (36.4\%) patients had sequelae and 1 patient was death.

Conclusions: Patients with SLE or SS with clinical features of NMOSD should be tested for AQP4-lgG. In our cohort, AQP4-lgG seropositive NMOSD arose in the context of low SLE activity and in the context of SS with extraglandular features and the disability and accrual damage at last follow up appeared to be mild.

\section{REFERENCE:}

[1] Wingerchuk DM, Banwell B, Bennet JL, et al. International consensus diagnostic criteria for neuromyelitis optica spectrum disorders. Neurology 2015 Jul 14;85(2):177-89.

Acknowledgements: No acknowledgements to report.

Disclosure of Interest: None declared

DOI: 10.1136/annrheumdis-2018-eular.6173 